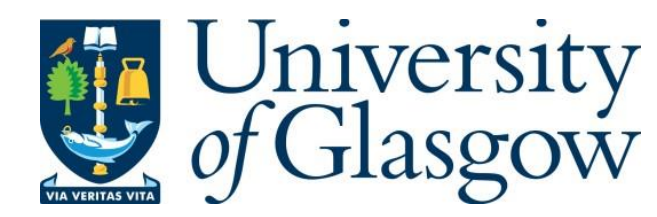

Doherty, C. (2019) Provoking knowledges and weaving conversations in teacher education. In: Nolan, K. and Tupper, J. (eds.) Social Theory for Teacher Education Research: Beyond the Technical-Rational. Series: Social theory and methodology in education research. Bloomsbury Academic: London, pp. 185-203. ISBN 9781350086395 .

There may be differences between this version and the published version. You are advised to consult the publisher's version if you wish to cite from it.

http://eprints.gla.ac.uk/195468/

Deposited on: 11 September 2019

Enlighten - Research publications by members of the University of Glasgow http://eprints.gla.ac.uk 
Provoking knowledges and weaving conversations in teacher education.

Catherine Doherty

School of Education, University of Glasgow

St Andrew's Building, 11 Eldon Street

GLASGOW G3 6NH UK

+441413303427

catherine.doherty@glasgow.ac.uk\# 


\title{
Provoking knowledges and weaving conversations in teacher education.
}

\author{
Abstract: The common-sense binary between "theory" and "practice" in teacher \\ education fuels unnecessary either/or arguments and adversarial models of teacher \\ education. This chapter will build a theoretical argument to reconcile these essential \\ domains of teachers' knowledge. It will highlight how the mutual provocation they \\ offer each other is an asset to harness in teacher education programs. The argument is \\ developed through three theoretical layers. Firstly, de Certeau's (1984) work dignifies \\ the generative improvizations and tactical creativity inherent in everyday practice, \\ thus how practice will inevitably and profitably exceed any theory thereof. Secondly, \\ Bernstein's (2000) sociology of education explains processes and relations between \\ knowledges in pedagogic discourse through the concepts of recontextualization, rules \\ of recognition/realization, and vertical/horizontal knowledge structures. Thirdly, \\ Bhaskar's (2002) meta-theoretical premise of “emergence" explains how theory and \\ practice interact to realize certain conditions and actualities in the complex, open \\ system of schools. A theoretical frame synthesizing these concepts will then be used \\ to articulate the logic and design underpinning the University of Glasgow's \\ partnership model for teacher education placements in both primary and secondary \\ school settings. This model stages three distinctive conversations amongst pre- \\ service teacher, university tutor, and mentor teacher: discussion following \\ observation of a peer's practice; embedded seminars; and joint assessment of the \\ placement. The design behind these encounters will be analysed to demonstrate how \\ theory and practice are catalytic, bringing different kinds of players and their \\ knowledges together to provoke generative professional insight.
}




\section{Focus on theory}

This chapter will highlight the mutual provocation that decontextualized theories and contextualized practices offer each other, and how such provocation is an enriching asset to harness in teacher education programs, particularly the practicum. The argument is developed through three theoretical layers. Firstly, de Certeau's (1984) work dignifies the generative improvizations and tactical creativity inherent in everyday practice, using the resources available in the setting in protean ways. Secondly, Bernstein's (2000) sociology of education explains processes and relations between knowledges in pedagogic discourse. The concept of recontextualisation highlights the space of freedom in any pedagogic setting for local context and agents to exert their influence. The concept of vertical and horizontal knowledges describes how different knowledges can co-exist - either stacking up into generalised truths or spreading out across different fields of contextual specificity. This distinction characterises the necessary tension when applying theoretical maxims to the local conditions of "thisness" (Thomson 2002). Thirdly, Bhaskar's (2002) meta-theoretical premise of "emergence" explains how theory and practice interact in the complex, open system of schools where different potentials compete to be realized. Cazden's idea of "weaving" offers a way to accommodate and harness the different contributions of theory and practice and to bring them into conversation with each other. Each of these steps is developed in more detail below.

A theoretical frame synthesizing these layers is then used to articulate the design underpinning the University of Glasgow's partnership model for teacher education placements in both primary and secondary school settings. This model stages three distinctive conversations amongst pre-service teacher, university tutor, and mentor teacher: discussions following observations of a peer's practice; embedded seminars; and joint assessment of the placement. These encounters demonstrate how theory and practice can be 
catalytic, bringing different kinds of players and their knowledges together to provoke professional insight. The conclusion reflects on how such a model that seeks to actively integrate the domains of theory and practice might inform research, and be pushed and challenged.

\section{Theory and practice as entangled}

Teacher education has long been plagued by a common-sense binary between "theory" and "practice" that fuels unnecessary either/or arguments and adversarial models of teacher education. A slogan of partnership has been embraced in recent teacher education reforms as a pragmatic and politically palatable way to "bridge" what is typically termed the "practice and theory divide". Like "community", and "motherhood", the word "partnership" comes with a warm glow that suggests it is a good thing, in and of itself. This label is doing normative work and its morally infused status makes any criticism or scepticism difficult. There are however different degrees and numerous models of partnering between the school as workplace and the higher education setting (Maandag et al. 2007). In Scotland, the influential Donaldson report (2011) recommended "a more integrated relationship between theory and practice, between the academic and the practitioner, between the provider of teacher education and the school" (p. 4). Rather than a strict division of labour allocating "theory" to universities and "practice" to schools, Donaldson characterized these partnerships as "relationships which are collaborative rather than complementary" (p. 7). This stance asks educational theory and practice to dance together, not just pass the relay baton for school placements.

An analytic distinction between the concepts of theory and practice can be useful at times. However, leaving the common-sense dualism unexamined can naturalize and reify the distinction as some kind of antagonistic opposition and mutual incompatibility. The relation has long been understood to be more complex and entangled. On a broad scale, Dewey 
described the "organic" relation between theory and practice that "enriched" learning processes:

... children bring the experiences, the problems, the questions, the particular facts which they have found, and discuss them so that new light may be thrown upon them, particularly new light from the experience of others, the accumulated wisdom of the world - symbolized in the library. Here is the organic relation of theory and practice ... getting from the start some intellectual conception that enters into his practice and enriches it. (Dewey [1902]1990, 85)

Durkheim considered the relationship between theory and practice in the field of education to be uniquely integrated: "educational theories have the immediate aim of guiding conduct. ... Their raison d'etre is in action. It is this dual nature that I have been trying to express in referring to education as a practical theory ... It is not action itself and thus cannot replace action. But it can provide insight into action" (Durkheim [1925] 1973, 2). Durkheim constructed this relationship between theory and practice as one of necessary symbiosis: "If educational theory goes beyond its proper limits, if it pretends to supplant experience, to promulgate ready-made formulae that are then applied mechanically, it degenerates into dead matter. If, on the other hand, experience disregards pedagogical thinking, it in turn degenerates into blind routine or else is at the mercy of ill-informed or unsystematic thinking” (Durkheim [1925] 1973, 2).

More recently Eisner (2002) and others (for example, Kinsella and Pitman 2012, Korthagen and Kessels 1999) have reanimated and appropriated Aristotle's philosophic distinction between episteme ("true and certain knowledge") (Eisner 2002, 375) and phronesis (“a kind of morally pervaded practical wisdom”) (p. 381). These terms better describe the condition of knowledge in teacher education, and the field's futile aspiration to certain, value-free science or episteme. Rather, Eisner argues, the field relies on the practical 
and moral reasoning of phronesis: "Practical reasoning is deliberative, it takes into account local circumstances, it weighs tradeoffs, it is riddled with uncertainties, it depends upon judgement, profits from wisdom, addresses particulars, it deals with contingencies, is iterative and shifts aims in process when necessary" (p. 375). This interpretation of the ancient concept of phronesis would thus dignify the particularity of contexts, situations and students, and the press of moral constraints and priorities (see critique by Kristjansson 2005). Such wisdom, Eisner argues, is accrued through experience over time, along with a level of artistry and aesthetics of teaching.

Beyond education, De Certeau's (1984) theory of everyday practice as "ways of operating" (p. xiv) offers an intriguing and playful concept of practice. Practice "is devious, it is dispersed, but it insinuates itself everywhere, silently and almost invisibly" (p. xii). Practice is creative and productive, in the opportunistic "ruses" (p. xv) and "mutations" (p. xxi) made by individuals in the moment, and in the makeshift "making do" with the institutional resources, rules and systems to hand. De Certeau dignifies these as a generative layer of appropriation, improvisation, and re-use: "users make ... innumerable and infinitesimal transformations of and within the dominant cultural economy in order to adapt it to their own interests and their own rules" (de Certeau 1984, xiii-xiv). These "tactics of practice" (p. xvii) are important resources in themselves, being innovative, disruptive or subversive contributions that warrant attention. Under this lens the user/practitioner adds value and extends possibilities - practice will always exceed the imagination of theory in unforeseen ways.

From these versions of how theory and practice relate to each other, I take the understanding that both dimensions matter in our field, and that teacher education profits from looking both ways like cock-eyed chameleons. While each serves as a necessary adjunct to the other, it is also a provocation or irritant. This is a more dynamic and dialogic 
relationship than models that would neatly derive practice from theory, or distil theory from a sampling of practice. For this reason, debates that pit "theory" against "practice" in teacher education as some kind of zero-sum game are reductive and unhelpful.

\section{Bernstein's sociology of knowledge}

To flesh out this relation of mutual provocation and generative dialogue to characterize school/university partnerships in teacher education, I now turn to Bernstein's work in the sociology of education and the sociology of knowledge, in particular his concepts of recontextualization, rules of recognition and realization, and knowledge structures. This network of concepts can illuminate any pedagogical relationship, scale or setting, including teacher education.

The concept of recontextualization explains how plans and rules never quite unfold according to the original plan. Bernstein (2000) was interested in pedagogic discourse as the social mechanism whereby knowledge produced in the academy or skills developed in industry were reconstituted for educational settings and ultimately distributed. By recontextualization, he is referring to the process whereby knowledge, skills, or discourses are taken from their original context of production into another context for pedagogical purposes. His key insight is that with any process of recontextualization, there will be some degree of transformation:

As the discourse moves from its original site to its new positioning as a pedagogic discourse, a transformation takes place ... because every time a discourse moves from one position to another, there is a space in which ideology can play. No discourse ever moves without ideology at play. As this discourse moves, it is ideologically transformed; it is not the same discourse any longer. (Bernstein 1996, 46) 
In other words, the "discursive gap" that opens whenever knowledge is recontextualized gives every player along any chain of curricular (or policy) implementation an opportunity and degree of agency to insert their own flavour and preferences into the process. This space of potential transformation resonates with de Certeau's more generalised notion of playful improvization in everyday practice and tactical appropriation of what the official order offers.

Teacher education without recontextualization would amount to directing students to a library of primary texts. Similarly, "teacher-proof" materials would seek to limit and deny the profession's work of recontextualization. Teachers' work inherently involves the work of selecting, translating, adapting and curating knowledge for students - be they school students, kindergarteners or teacher education students. For this reason, when other parties come to the table, new versions, new priorities and new ideologies will inevitably enter the conversation. For teacher education, with its multiple sites, disciplines and partners, recontextualization helps to explain a lot of the "slippage" and contradictory advice our teacher education students encounter both within the higher education setting and in the school workplace. Is that necessarily a bad thing?

If the field of teacher education works on the assumption that there is ultimately a universal science to learning and teaching (an "episteme"), then the proliferation of such diversity or slippage would be baffling and counterproductive. If the field works on the assumption that learning and teaching are complex open-ended processes, involving and cultivating "phronesis" over time, then such diversity becomes enriching and stimulating food for thought. In teacher education, student teachers move across contexts, and work with a variety of knowledgeable others. Teacher educators cannot, and should not, expect access to tightly sealed heads into which uncontaminated truth can be uploaded, as depicted in "The Matrix". The hard won case for teachers' professional status stems from the need and the capacity to work in a problematic knowledge space, juggling competing priorities and 
negotiating dilemmas rather than performing predictable, technical routines. The work of recontextualization is inevitable, inherent, creative and generative.

Bernstein also makes a useful distinction between what he calls the "recognition rule" and the "realization rule" (2000). The recognition rule refers to the knowledge required to appreciate how special requirements, discourses and protocols mark a context and "orientates the speaker to what is expected, what is legitimate to that context" (p. 17). The realization rule refers to the knowledge required to produce these discourses and enact such protocols in a validated way. This distinction helps us think about the school placement as a learning experience. The preservice teacher will learn to "recognize" the settled productive classroom, the engaged student, or the competent teacher, but it takes a different mode of knowing and greater degree of understanding to unpack and learn how such desirable qualities have been accomplished, or "realized". Judging certain practices to be good is still a long way from being able to reconstitute those practices oneself. Surface mimicry relying on knowledge of the rules of recognition alone will not be enough. There are deeper relations and forces producing the classroom performance.

In linguistics a similar concept of "realization" captures how particular wording choices in speech or writing are understood to "realize", or bring to fruition, the deeper metafunctions of language, these being the semantic field, relational tenor and the mediating mode of any text (Martin 1992). This theory allows linguistic analysis to delve below the surface of any text to ask different kinds of questions: "so when we analyse a text, we show the functional organization of its structure; and we show what meaningful choices have been made, each one seen in the context of what might have been meant but was not" (Halliday and Matthiessen 2004, 24). This question of "what meaningful choices have been made ... in the context of what might have been ... but was not" could equally inform an analysis of classroom practice. This question would open up conversations about competing 
possibilities, decision pathways, judgement calls, recontextualization, improvisations, teacher thinking and pedagogic design, and foreground the cultivation of phronesis.

In the linguistics community, the idea of "realization" is often explained metaphorically through the relationship between weather and climate: today's weather is a realization of the larger climate system. Again, this offers a helpful way to think about how teachers' practice "realizes" theories of learning/teaching, whether or not those theories are explicit. Tacit notions of good practice rely on guiding principles that can implicitly apply across particular contexts. Such theoretical precepts, templates and the culture of expectations built around them could be considered to constitute the "climate" in any educational setting. Teachers' work is framed and only possible within this larger context of policies, thought, conventions and expectations. A teacher will tactically "realize" particular practices within the culture, constraints and enablements of their particular settings, as well as through their own ideological filters in the process of recontextualization.

\section{Bhaskar's metatheory for open systems of competing potentials}

By invoking terms like "surface" and "deeper potentials", I am drawing on the critical realism of the late Roy Bhaskar, in particular his principle of emergence in a layered ontology. Bhaskar's philosophy of social science accounts for a complex social world, one in which both the "intransitive" material world and its "transitive" discursive politics create effects and possibilities for people and their actions. For Bhaskar, reality comes about through a process of realization or "emergence" being the outcome of the interactions between underlying potentials.

For Bhaskar (2002), social reality stems from a deep layer of potentials and capacities that are inherent in actors, objects or systems. The climate system, with its complex underpinnings in the potentials of tides, air pressure systems and ocean 
temperatures, serves as a useful metaphor again. Similarly, schooling is premised on disciplines, policies, parties, and philosophies with each dimension projecting and priming certain possibilities. These multiple potentials will either cohere or compete within an "open system" (p. 26), and from this contingent mixing of forces and the tactical ruses of teachers, certain actualities will emerge ${ }^{\mathrm{i}}$. Only some of these actualities will ultimately be evident at the empirical surface (what can be observed, sensed, or measured), while other possibilities may never eventuate, or may not surface empirically. In schools, this distinction is exemplified in the learning that is rendered empirically visible by assessment practices, and the learning that is less observable and measurable, but nonetheless real. This meta-theory demands equal attention to what is not present, what does not emerge, or what is not evident, and consideration of how certain possibilities may have been stymied or suppressed by complicating and competing factors. The contemporary appetite for "evidence-based" approaches fails to dignify this complexity.

For Bhaskar, the question to ask of any empirical phenomenon is what underlying premises needed to be in place for it to have emerged uses a similar term. Bhaskar used the social example of Christmas dinner to demonstrate all the preceding social layers that allowed this particular social practice and its moment at the festive table to emerge. We could equally use the example of a particular practice observed in a classroom, and ask "what are all the social layers that have gone into producing this practice or moment?" This forces any analysis of social phenomenon (including that of classroom practice) to find ways to look below the empirical surface, to enquire into pre-existing factors, counterforces, enablements and constraints. If current theoretical thinking promotes certain kinds of practice, why doesn't such "best" practice simply emerge? What other factors might be at play here, mitigating against such sponsored practice? 
Bhaskar's layered ontology can accommodate Bernstein's concepts of "rules of realization”. Both point to deeper conditions that allow a practice to emerge or be realized, and any particular "recontextualization" to be understood as outcome of the play of forces and conditions of possibility in an open system. It can also dialogue with de Certeau's opportunistic and creative form of practice that profits from the potentials at hand to suit the actor's purpose.

With this kind of layered logic, we can build a more complex understanding of what practice we see on the surface, in terms of what goes into allowing it to emerge. We can also appreciate how new policies and practices cannot simply be implemented with perfect fidelity when added into complex systems of competing potentials. Rather, various realizations of their design for practice will emerge from the competition between enabling and constraining conditions and other confounding forces such as the contradictory tangle of policy and curricular obligations teachers are often dealing with at any point in time. Any practice that emerges on the surface will have been filtered and adjusted through each agent's work of recontextualization and operational tactics in this complex open system. This is why and how theory and practice knowledges cannot be neatly conflated, but tend to irritate and provoke each other in a generative dialogue.

\section{Bernstein's knowledge structures}

Bernstein's (2000) distinction between horizontal and vertical knowledge structures offers another dimension on which to map the different types of knowledge that contribute to theory/practice dialogues in the field of education, and understand how they relate to each other. For Bernstein, a vertical knowledge structure is one that stacks up in a principled fashion, such that the pieces or contributions of knowledge build to an axiomatic apex that can illuminate all layers below. The abstract heights of theory in pure maths or physics might 
best exemplify this - the more abstracted and decontextualized the theory, the more it explains. The top level integrates or subsumes lower level propositions. Bernstein then used the term "vertical discourse" to refer to the specialized language used to express such abstracted vertical knowledge. Vertical discourse uses theoretical terms and this specialized language becomes a crucial part of the vertical knowledge structure. These languages are powerful resources for those who have access to them, but will exclude others who do not speak that language. If one educational theory could account for all educational practice, then we might be able to construct teacher education programs using a vertical knowledge structure, and help our graduates into speaking a coherent vertical discourse. First year would logically prepare for second year, and so on in a lock step fashion, creating a coherent vertical stack.

In contrast, in a horizontal knowledge structure, bodies of knowledge sit beside each other as parallel universes, knowing and representing the same objects of study in different and incompatible ways. Given their different discourses and logics, these knowledges cannot be assembled into an integrated hierarchy. Rather these bodies of knowledge remain discrete and distinct, each cultivating its own perspective. Bernstein refers to this arrangement as “segmental" (2000, p. 157), like the pieces of an orange.

Given the different disciplines feeding into the field of educational thought, teacher education programs tend to reflect more this horizontal knowledge structure, though perhaps with aspirations towards building vertically. By this, I mean we do not have one allconsuming abstract theory to explain all educational processes, but we do have multiple theoretical resources, each with their own degrees and languages of abstraction.

Bernstein considered the field of education to be a "region" of horizontally organized knowledges. By this, he meant that as a field of study serving a profession, it occupies a busy 
intersection between multiple informing disciplines that speak different conceptual languages. Under this lens, the struggle in our field is not between theory and practice; rather it is between multiple theories (historically sampling sociologies, psychologies, histories and philosophies). These different disciplinary gazes and their technical vocabularies or vertical discourses may or may not conflict in their meanings, and may or may not emerge in the realization of practice at the empirical surface. Practice thus serves as the integrating device or selective mechanism through which each discipline and its form of wisdom might contribute part of the story. This is neither a good thing nor a bad thing - it is the nature of the beast.

For example, behaviour management or classroom discipline is an important object of study treated under multiple disciplines in teacher education programs (Hayes and Doherty 2017). In psychology, there will be different theoretical discourses that seek to conceptualize problems and solutions in behaviour management, such as operant conditioning, motivation theory or Kohlberg's moral stages. Sociological theory might frame behaviour management in terms of engagement, resistance, marginalization, or curricular relevance. Philosophers might refer to disciplinary society's self-regulation or questions of individual autonomy. Neuroscience may start contributing in this space soon enough. The different premises underpinning each vertical discourse mean that teacher education programs cannot stack these theoretical resources into one coherent vertical structure for students. Rather we guide them across the horizontal field of possible approaches, each with its own vertical discourse and theoretical abstractions ${ }^{\text {ii }}$.

Bernstein then described what he termed "horizontal discourses". In contrast to the de-contextualized abstractions of vertical discourse, horizontal discourses are highly contextualized ways of speaking that come from everyday contexts, typical in "face to face encounters, where meanings are likely to be both context specific and independent" (2000, p. 
208). Context demands this specificity. While vertical discourse would strip context from its theoretical abstractions, horizontal discourse is "context specific and context dependent" (p. 157). This is where "tips" of practitioner wisdom operate - in the immediately accessible plane of contextualized action. For example, “Don’t smile till Christmas!'”iii is a notorious behaviour management tip for the pre-service teacher. This guidance could be expressed or explained in cognate vertical discourse, for example in terms of Bernstein's (1971) concept of stronger or weaker framing of the teacher/student relations, but when expressed in the horizontal discourse, it speaks efficiently to the context and its immediate demands.

Thomson's (2002) concept of "thisness" captures this necessary shift to pay attention to the lived particularities of school contexts, particularly the schools of post-industrial communities:

Rustbelt school administrators, teachers, parents and students routinely begin their sentences saying "This school ... these kids ... this community ...” In order to understand thisness, it is necessary to think of the school as a particular material place. Each school "place" is a distinctive blend of people, happenings, resources, issues, narratives, truths, knowledges and networks, in and through which the combined effects of power-saturated geographies and histories are made manifest. .. The school as a place is embedded in context and cannot be detached from it. (pp. $72-$ 73)

“Thisness" demands attention to the confounding factors, circumstances and forces that distinguish any school context. Thisness refuses the theoretical generalization. This distinction between vertical and horizontal discourses explains the gear change that students experience as they move from campus studies into the school placement setting. In the former, they are talking about generalized ideas and principles - that which might be 
abstracted from context. In the latter they are talking about a particular setting and its demands - that which cannot be ignored in the here and now.

These different structures of knowledge and shifts in discourse need not be cast as a problem in teacher education. Courtney Cazden's recent work explored how the different types of knowledge become complementary resources which, in their interplay, contribute more than the sum of their parts. Cazden has sustained a close interest in classroom discourse over a long history of changes in pedagogic ideologies, and across diverse communities. She developed a concept of "weaving" in a large project assessing the quality of classroom practice in Singapore. The metaphor of weaving "names the moments in classroom lessons when explicit connections are made - by teachers or students - across one or another dimension of knowledge. Usually weavings connect something that is already familiar with new curriculum content" (Cazden 2006, 1). Cazden condenses this down to the phrase "connected learning". She cites a similar argument in the work of Dewey, regarding "the best type of teaching" as that which "puts the students in the habitual attitude of finding points of contact and mutual bearings" (citing Dewey, p. 3). Cazden lists a variety of cognate "crossconnections" that other theorists have advocated, then develops a typology of how connections can do cultural, cognitive or critical work. She gives examples that range from incidental moments, to extended learning sequences of purposeful weaving across different "ways of knowing" (p. 15).

This concept of "weaving" offers a generative way to capture how talk in teacher education shuttles between vertical and horizontal discourses. We jump from concept to concrete example and back again. We also move across different horizontal discourses to draw connections between examples that offer particular moments in time or different settings. This idea of weaving can be adapted to purposefully and mindfully craft partnership conversations between university staff, preservice teacher and on site mentor teachers. Such 
conversations build connections across theories (plural), between a theory and its realization or recontextualization in practice, or between contexts of practice. The metaphor of weaving implies a repeated going to and fro - repetitive action that crafts a larger fabric of unique texture. Our task in teacher education partnerships becomes one of making this kind of mindful weaving habitual in the professional dialogues between parties, and making the fabric it builds one that is robust and durable but also flexible.

Thus far, this paper has developed a vertical discourse using de Certeau's concepts of tactics and improvisations, Bernstein's concepts of recontextualization, rules of recognition and realization, vertical and horizontal knowledge structures, vertical and horizontal discourses, Thomson's concept of thisness and Cazden's concept of weaving to think about the relations between theories and practice in teacher education. In the next section, I profile the three signature conversations that have been designed and embedded in the University of Glasgow's partnership model for teacher education. I use the vertical discourse assembled developed as a way to extract aspects of the design that others might find pertinent for their contexts.

\section{Weaving a partnership in teacher education}

Historically, teacher education in Scotland involved a high stakes routine known as "the crit", whereby a university staff member visited the teacher education student on placement once to observe and evaluate a lesson and thus assess the student's progress. In marked contrast, the University of Glasgow's current partnership model for teacher education aims to nurture and enrich ongoing mutual exchange between university teacher educators, the teacher education student and their school mentors - those who Livingston (2014, 219)calls the "“hidden' or 'unrecognized' teacher educators." This intense investment in cultivating collaborative 
partnerships between universities and schools distinguishes this model from the school-based teacher "training" models now influential in England (Brown 2018).

The model orchestrates three signature conversations during the school placement: the onsite seminar, the learning observations and the joint assessment visit. The design hinges on the embedded university tutor facilitating relationships. However, with repeated comings and goings across institutional boundaries, their role as "boundary brokers" (ET2020 Working Group on Schools Policy $(2014 / 15)$ 2015, 38) also serves as a provocation and irritant that stirs up different types of conversations. The conversations about professional practice precipitated amongst the teacher education students themselves are another feature and resource in the design. The program of rolling conversations between students, mentoring teachers and university tutors weave theory and practice into a strong fabric of shared professional dialogue, much more so than the historical "crit" and its power imbalance could.

The regular onsite seminars stage joint conversations between the university tutor and the group of students allocated to a geographical cluster of schools including both primary and secondary settings. This sectoral mix and the rotation around school settings are purposeful and generative features of the model. Each seminar sets an agenda around a generic aspect of teachers' practice (for example, questioning, planning, assessment, and positive relationships) (School of Education 2017) pushing students' understanding towards a more decontextualized vertical discourse. The seminar topics are "responsive to the needs of the local school community to draw upon expertise amongst school staff”, and school mentors are invited to participate and share their "view from practice" (p. 18). By bringing student teachers together from different years, subjects and school settings, contextualized examples of the generic focus can be pooled, and connections can be woven between the horizontal "thisness" of their varied settings and the vertical discourse of generic principles or theory. These conversations are equally opportunities where students can articulate the 
culture and constraints that shape practice in their setting. In this way, the "open system" and complex realities of schools that students are immersed in can be dignified and analysed.

Students are given a window on the "thisness" of each other's settings, and benefit from each other's worked examples in their understanding of what is shared and what is distinct across settings.

The learning observations create opportunities for the student teachers in each cluster to observe aspects of each other's practice. These observations and the following de-briefing discussion between observing students, the university tutor and the host mentor teacher will focus on the generic topic/issue raised in the seminars. There is a strong resonance between these peer observations and Gore's more comprehensive design of quality teaching rounds (Gore et al. 2015) for teachers' continuing professional development. Again, the design pays close attention to purposefully crafting the conversation that follows these shared observations, to help the student teachers process what they see and articulate what they think: "The focus of this is on practice in relation to the theme and at no time is the observed student evaluated or discussed. The key focus is the use of this professional dialogue to improve one's own practice."

These terms of engagement are very explicit - the conversation is not about evaluating the other student's practice. Rather, the point is to use the shared, contextualized observations to provoke and reflect on one's own choices and professional thinking in similar circumstances. Suspending judgement is harder than it sounds, and students need constant support from the university tutor to use the observation as a springboard for reflection on their own practice and preferences.

The weaving achieved here is across contexts. This design pushes the student from attending just to the surface rules of recognition to considering deeper rules of realization from which the observed practice emerged. The students are challenged along the same lines 
as that generative linguistic question: "what meaningful choices have been made $\ldots$ in the context of what might have been ... but was not" (Halliday and Matthiessen 2004, 24). It helps the observing teacher education students appreciate the multiple competing potentials in classrooms that may or may not play out given other contextual factors.

The observed student's mentoring teacher attends these post-observation conversations when possible, and can bring knowledge of the contextual "thisness" that was not empirically evident to the student observers. This kind of encounter also broadens the base of knowledgeable others the students are exposed to in each setting, and offers more interpretations of what they observed. The participating teachers and tutors can also benefit from having their thinking provoked. It will be in these conversations that the student teacher will both cultivate and display the capacity to negotiate the gear shift between the horizontal discourses of "here", and the vertical discourses available to the profession.

The joint assessment visit is the culminating conversation between the mentoring teacher, and the university tutor to collaboratively construct the final evaluative report on the student's placement. Given the preceding conversations in the seminars and learning observations, there will have been formative discussions including the student across the learning journey, and opportunity for the university tutor and the school mentor to develop a shared understanding and language around what counts in evaluating practice. In contrast to the isolated "crit" visit with its imposition of external criteria, and the hit and miss of what lesson was observed, all parties in this partnership model have engaged in informed dialogues and constructive reflections along the way. The co-construction of the report is perhaps the strongest evidence of a working partnership, and mutual consideration between representatives of the domains of theory and practice. The evaluation aligns with the General Teaching Council of Scotland's Standard for Provisional Registration (2017) which anchors 
these conversations in the broader profession's shared agreements around deep principles and potentials.

As a curriculum design, this model favours neither theory nor practice. Rather it creates attention to the relations and "meaningful tensionality" (Aoki cited in Lee 2017, 25) between the two domains. It harnesses their contributions of expertise in a generative dialogue that weaves between vertical discourses and contextualized particularities, and invites the students to observe and understand a number of horizontally organized contexts. The field of education is oversupplied with theoretical resources and approaches, and school settings are complex open systems where the conditions of possibility shape choices and emergent outcomes. Teacher education needs to cultivate a professional disposition that can navigate and profit from this complexity.

\section{Focus on theory}

This chapter has conceptualized the relationship between the theoretical ideas and grounded wisdom encountered in teacher education, then the design of partnerships that might bring these differently textured knowledges into a productive conversation with each other. To this end the chapter assembled a selection of theoretical and meta-theoretical resources to craft a more catalytic relationship between the theoretical knowledges and the phronetic wisdom of practice that contribute to teacher education programs. Teacher education is often caricatured as occupying "the mine-strewn border between theory and practice" (Labaree 1996, 41). I have argued that these domains of expertise can be productively understood and profitably harnessed as mutual provocations in robust partnerships for teacher education.

Teacher education enjoys an embarrassment of riches: firstly in its theoretical resources from multiple disciplines; secondly in the mutable capacity of practice to exceed and escape theory; and thirdly in its necessary embedding in the constitutive "thisness" 
(Thomson, 2002) of any context. De Certeau's (1984) concept of everyday practice dignifies and values the creative ruses and improvizations that make do, solve problems and get jobs done. Bernstein's concept of recontextualization similarly highlights the ever present opportunity for generative agency in the knowledge work of pedagogues. However, the value-addedness these theorists highlight tends to be overlooked or erased in research accounts because of its ephemeral and contingent nature. There is a challenge here for research to capture the circumstantial happenstance of practice.

Bernstein's distinction between rules of recognition and rules of realization explains how observations of practice can dig deeper, to understand the conditions of possibility, expectations and constraints of any context. This appeal to deeper systems to explain surface events or absences was further framed through the process of emergence amongst competing potentials, as outlined in Bhaskar's critical realist philosophy of social science. Teachers will recognize this dynamic and layered complexity more than they would the sterile promises of decontextualized "best practice". These deeper conditions and potentials may not be empirically observable, but are nevertheless integral to understanding what happens in classrooms.

Bernstein's concepts of vertical and horizontal knowledges helped to map the condition of knowledge in teacher education, being one with limited capacity to build vertically, but rather a tendency to spread horizontally collecting multiple theoretical languages. The grounded, horizontal discourse of practice serves as the integrating device through which different theoretical stances compete for relevance and expression. Cazden's concept of weaving offered a metaphor for how discourse around educational theories, practice and contexts can be drawn into the same rich pedagogic conversation. The signature conversations of the Glasgow Partnership Model offer an example of a curricular design that stages provocative conversations that weave theoretical knowledges and practice insights 
together for the teacher education student. The weaving between vertical and horizontal discourses and across contexts can test congruence and expose disjuncture, preparing the teacher education student for a profession positioned at a busy intersection of interests, theories and politics. The wealth of possible theories may pose a problem as much as a resource for researchers.

In terms of caveats on this design for teacher education partnerships, these kinds of engaged conversations and mutual trust take time to cultivate. Programs in a rush to produce teachers may not furnish the necessary space and time for such interactions. Similarly, this model will not deliver the reductive certainties of "mechanistic" (Doll, 2013, 194) technicism or the "robotization of teaching" (Aoki, cited in Lee, 2017, 20), but rather will cultivate a disposition to consider contextual conditions, local constraints and theoretical possibilities as important and necessary factors in pedagogic work.

\section{References:}

Bernstein, B. (1971), 'On the classification and framing of educational knowledge', in M. Young (ed), Knowledge and control: New directions for the sociology of education, 47-69, London: Collier Macmillan.

Bernstein, B. (1996), Pedagogy, symbolic control, and identity: theory, research, critique, Washington, D.C: Taylor \& Francis.

Bernstein, B. (2000), Pedagogy, symbolic control and identity (revised), Lanham: Rowman and Littlefield.

Bhaskar, R. (2002). From science to emancipation: Alienation and the actuality of enlightenment, New Delhi: SAGE.

Brown, T. (2018). Teacher education in England: A critical interrogation of school-led training, London: Routledge.

Cazden, C. (2006), 'Connected Learning: "Weaving" in classroom lessons', keynote presentation at Pedagogy in Practice 2006 Conference, University of Newcastle, NSW. Available online:

https://www.researchgate.net/profile/Courtney_Cazden/publication/245769594_Conn 
ected_Learning_Weaving_in_Classroom_Lessons/links/5643c05608ae9f9c13e11b3c. pdf (accessed 8 July 2018).

de Certeau, M. (1984), The practice of everyday life, Los Angeles: University of California Press.

Dewey, J. ([1902] 1990), The school and society, Chicago: University of Chicago.

Doll, W. 2013. 'Complexity and the culture of curriculum', Educational Philosophy and Theory, 40 (1): 190-212.

Donaldson, G. (2011). Teaching Scotland's future: Report of a review of teacher education in Scotland, Edinburgh: The Scottish Government.

Durkheim, E. ([1925] 1973), Moral education: A study in the theory and application of the sociology of education, trans. E. Wilson and H. Schnurer, New York and London: Free Press MacMillan.

Eisner, E. (2002), 'From episteme to phronesis to artistry in the study and improvement of teaching', Teaching and Teacher Education, 18: 375-85.

ET2020 Working Group on Schools Policy 2014/15, (2015), Shaping career-long perspectives on teaching: A guide on policies to improve Initial Teacher Education, Brussels: European Commission.

General Teaching Council of Scotland. (2018), Professional Standards 2017. Available online: http://www.gtcs.org.uk/professional-standards/professional-standards.aspx (Accessed 25 March 2018).

Gore, J., M. Smith, J. Bowe, H. Ellis, A. Lloyd, and D. Lubans. (2015), 'Quality Teaching Rounds as a professional development intervention for enhancing the quality of teaching: Rationale and study protocol for a cluster randomised controlled trial', International Journal of Educational Research, 74: 82-95.

Halliday, M., and C. Matthiessen. (2004), An introduction to functional grammar (3rd ed.), London: Arnold.

Hayes, D., and C. Doherty. (2017), 'Valuing epistemic diversity in educational research: An agenda for improving research impact and teacher education programs', Australian Educational Researcher, 44 (2): 123-139.

Kinsella, E. and A. Pitman. (2012), 'Engaging phronesis in professional practice and education', in E. Kinsella and A. Pitman (Eds), Phronesis as professional knowledge, 1-11, Rotterdam: Sense. 
Korthagen, F., and J. Kessels. (1999), 'Linking theory and practice: Changing the pedagogy of teacher education', Educational Researcher, 28 (4): 4-17.

Kristjansson, K. (2005), 'Smoothing it: Some Aristotelian misgivings about the phronesispraxis perspective on education', Educational Philosophy and Theory, 37 (4): 455473.

Labaree, D. (1996), 'The trouble with Ed schools', The Journal of Educational Foundations, 10 (3): $27-45$.

Lee, Y-L. 2017. 'Lingering on Aoki's bridge: Reconceptualizing Ted Aoki as curricular techno-theologian', Journal of Curriculum Theorizing, 31 (3):18-30.

Livingston, K. (2014), 'Teacher educators: Hidden professionals?', European Journal of Education, 49 (2): 218-232.

Maandag, D., J. Deinum, W. Hofman, and J. Buitink. (2007), 'Teacher education in schools: an international comparison', European Journal of Teacher Education, 30 (2): 151173.

Martin, J. (1992), English text: System and structure, Philadelphia: John Benjamins.

School of Education. 2017. Primary school experience partnership handbook, 2017-18. University of Glasgow.

Thomson, P. (2002), Schooling the rustbelt kids: Making the difference in changing times, Crows Nest, NSW: Allen \& Unwin.

\footnotetext{
'Doll's $(2013,199)$ treatment of chaos and complexity theory in curriculum studies uses a similar vocabulary to argue "we might be able to design curricula or instructional strategies where ideas interacting with other ideas will catalyse themselves to develop (create) not only other ideas but ones more adaptable to the issues or problems at hand."

ii In Leonard Bernstein and Stephen Sondheim's musical "West Side Story", the song "Officer Krupke" gives an entertaining exemplar of a horizontal knowledge structure, with each verse posing a different solution to "juvenile delinquency".

iii Christmas in the northern hemisphere, Easter in the southern hemisphere.
} 